\title{
Modeling of Radiation Fields in a Sub-Picosecond Photo-Conducting System
}

\author{
K. A. REMLEY ${ }^{\text {a }}$, A. WEISSHAAR ${ }^{a}$, V. K. TRIPATHI ${ }^{\mathrm{a}}$ and S. M. GOODNICK $^{\mathrm{b}, *}$ \\ ${ }^{a}$ Department of Electrical and Computer Engineering, Oregon State University, Corvallis, Oregon, USA 97331; \\ ${ }^{\mathrm{b}}$ Department of Electrical Engineering, Arizona State University, Tempe, Arizona, USA 85287
}

\begin{abstract}
FDTD and Monte Carlo methods are combined to simulate the terahertz radiation from a coplanar photoconducting structure. The simulation tool under consideration allows calculation of potentials, particle distributions, current densities, and the near field electromagnetic fields anywhere in the computational domain. To model the far field radiation, it is not efficient nor, in many cases, physically possible at present to use the FDTD technique directly because of the excessive computational burden. Techniques are discussed for modeling both the near field and the far field radiation. Computational results showing the far field radiation are in qualitative agreement with published experimental results.
\end{abstract}

Keywords: Terahertz radiation, subpicosecond systems, photo-conducting systems, FDTD, time domain analysis, computational modeling, electromagnetic radiation, far-field radiation, near-tofar-field transformation, Hertzian dipole

\section{INTRODUCTION}

Characterization of the electromagnetic radiation arising from ultrafast electronic structures whose dimensions approach the wavelength of operation is a subject of continued research. The design of terahertz radiators $[1,2]$ as well as applications such as electrooptic sampling [3], switching [4], and terahertz spectroscopy [5] all require an accurate model of the radiated electromagnetic field. In some simple cases the radiated field may be calculated directly or approximated from a known excitation. Alternatively, numerical techniques such as the finite-difference time domain (FDTD) method allow computation of the fields radiated from structures with arbitrary physical geometry or material composition. For an accurate portrait of the radiated fields, the open region simulation space used in the FDTD should be terminated with absorbing boundary conditions (ABCs).

\footnotetext{
* Corresponding author.
} 
$\mathrm{ABCs}$ eliminate reflections at the edges of the computational domain, allowing simulation of propagation in infinite free space.

The computational burden of the FDTD method increases with the dimensions of the physical simulation space, and thus becomes less efficient for calculations far from the source. The FDTD also suffers from numerical grid dispersion for large computational domains. Additionally, the larger the simulation space, the larger the computational burden involved in the $\mathrm{ABC}$ calculations. It is, therefore, desirable to use alternate techniques for determination of the far field radiation.

\section{FORMULATION}

\section{Simulation Tool}

The present work utilizes a simulation tool which has been developed at Oregon State University [6]. The tool couples a Monte Carlo particle simulator to either a Poisson solver or an FDTD electromagnetic solver to determine device characteristics such as potential, particle distribution, and current density anywhere in the computational domain. In the present case, the FDTD solver is used, enabling determination of the electromagnetic (EM) fields throughout the simulation space. The Monte Carlo simulator and the FDTD solver are coupled together in a leap frog manner, allowing continual updates of both the particle distribution and the EM fields, see Figure 1.

The Monte Carlo particle simulator allows stochastic solution to the Boltzmann transport equation for particle motion. Particle motion is modeled as a series of free flights subject to magnetic and electric forces. Motion is terminated by instantaneous, random scattering events. Random scattering times are generated using a random number generator and the calculated quantum mechanical scattering cross-section. Important scattering mechanisms for GaAs are included in a three band model.

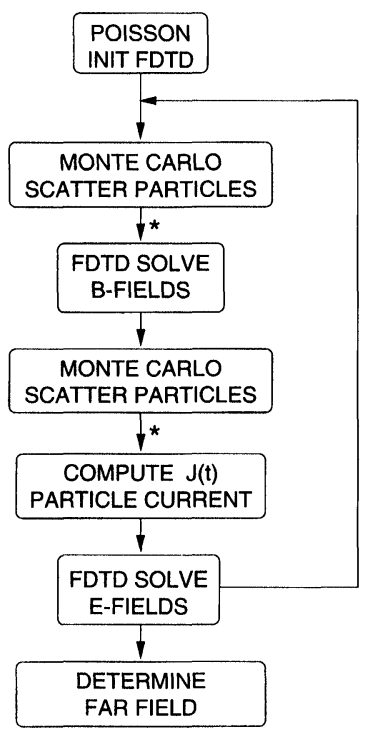

FIGURE 1 Computer algorithm used in the present work.

The FDTD scheme used in the present work allows modeling of complex, three dimensional structures. Centered differences are used in time and space, utilizing the Yee cell formulation [7]. This technique allows broadband response predictions and access to field components throughout the computational domain. The absorbing boundary conditions used in the simulations space are Berenger's Perfectly Matched Layer (PML) [8]. Impedance matching to a highly lossy medium is accomplished by utilizing electric and magnetic loss terms to attenuate only the outgoing portion of the wave. In the PML, absorption is theoretically independent of frequency and angle of incidence [8].

\section{Photo-Conducting Experiment}

The radiation arising from a photo-conducting system is modeled using the simulation tool described above. In this system, a sub-picosecond laser pulse incident on a GaAs substrate creates electron-hole pairs, as shown in Figure 2. Biased electrodes on either side of the pulsed area cause the electrons and holes to migrate away from each other, creating a dipole moment. A time-varying current density is induced in the GaAs substrate. 


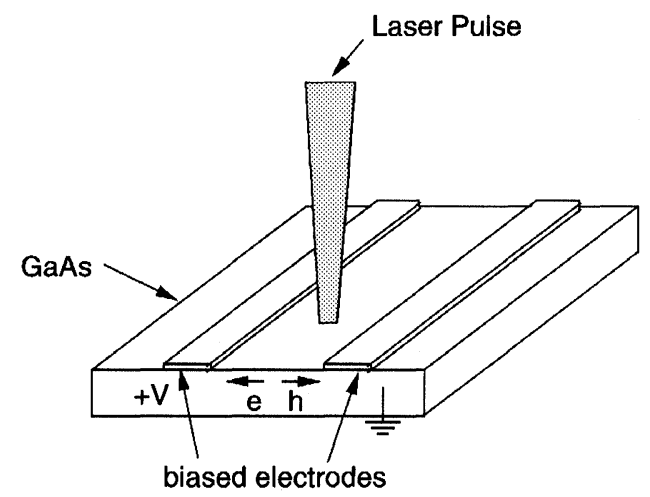

FIGURE 2 Typical structure for an electrooptic sampling experiment.

The rise time of the current pulse is extremely fast, on the order of a few hundred femtoseconds, as illustrated in Figure 3a. This current density is the source of the EM radiation in the photo-conducting experiment. Once the excitation is removed, the biased electrodes continue to attract the electrons and holes, therefore recombination occurs over a timescale much longer than the simulation time.

\section{RESULTS}

Figures $4 \mathrm{a}$ and $5 \mathrm{a}$ show the near field radiation along a surface above and parallel to the GaAs substrate of the structure shown in Figure 2. These fields were calculated using the simulation tool described above, with a bias of 40 volts on the metal electrodes, and an injection rate of $1 \mathrm{e} 15 / \mathrm{cm}^{3}$. In this simulation it was assumed that the laser pulse had a Gaussian wavefront with beam radius standard deviation of $2 \mu \mathrm{m}$, a Gaussian-distributed energy variation between $1.42 \mathrm{eV}$ and $1.62 \mathrm{eV}$ (larger than the bandgap of GaAs), and a pulse duration of approximately $20 \mathrm{fs}$.

If the source is modeled as a Hertzian dipole, i.e., a dipole with negligible length, the radiation may be found using the well-known equations for an infinitesimal dipole, repeated, for convenience, below. See e.g. [9], for the derivation.
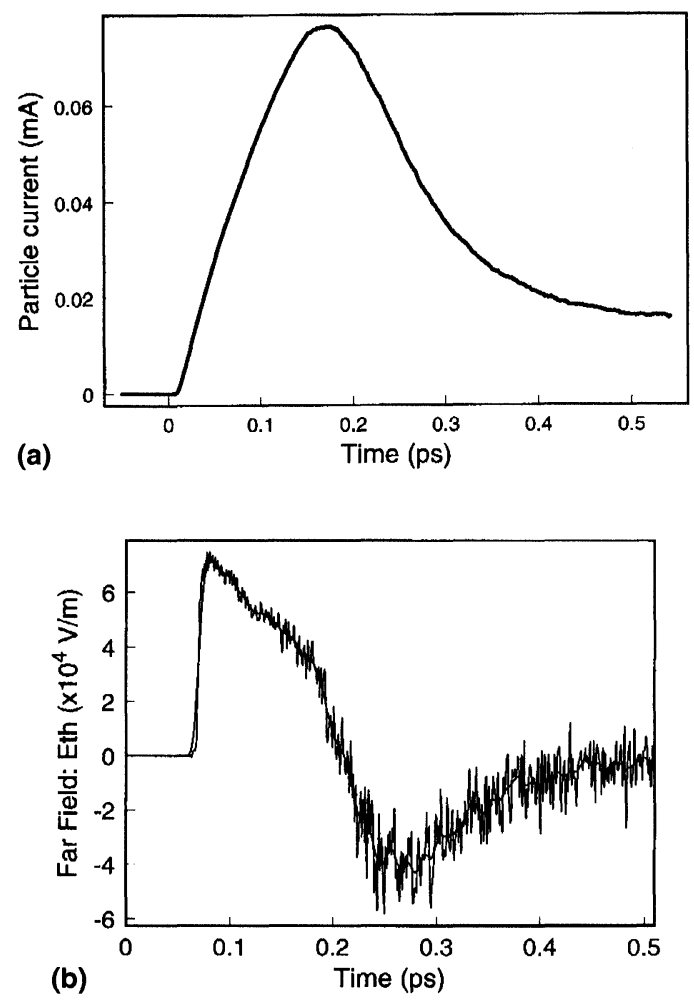

FIGURE 3 (a) Current density in the GaAs substrate for an injection rate of $1 \mathrm{e} 15 / \mathrm{cm}^{3}$ and bias of 40 volts. (b) Numerically smoothed time derivative of the current pulse shown in (a).

$$
\begin{aligned}
& \vec{E}(R, t)= \\
& \hat{a}_{R}\left[\frac { 2 \operatorname { c o s } \theta } { 4 \pi \varepsilon _ { 0 } } \left(\int_{t} \frac{\int_{v} J\left(\tau-\frac{R}{c}\right) d v^{\prime}}{R^{3}} d \tau\right.\right. \\
& \left.\left.\quad+\frac{\int_{v} J\left(t-\frac{R}{c}\right) d v^{\prime}}{c R^{2}}\right)\right] \\
& -\hat{a}_{\theta}\left[\frac { \operatorname { s i n } \theta } { 4 \pi \varepsilon _ { 0 } } \left(\int_{t} \frac{\int_{v} J\left(\tau-\frac{R}{c}\right) d v^{\prime}}{R^{3}} d \tau\right.\right. \\
& \left.\left.\quad-\frac{\int_{v} J\left(t-\frac{R}{c}\right) d v^{\prime}}{c R^{2}}+\frac{\frac{\partial}{\partial t} \int_{v} J\left(t-\frac{R}{c}\right) d v^{\prime}}{c^{2} R}\right)\right]
\end{aligned}
$$

Here $R$ is the distance from the dipole to the far field observation point, $J$ is the volume current density induced by the dipole, and $\theta$ is measured off the dipole axis. Figures $4 \mathrm{~b}$ and $5 \mathrm{~b}$ show the fields from an ideal dipole for comparison to that of the near field radiation found using the FDTD 

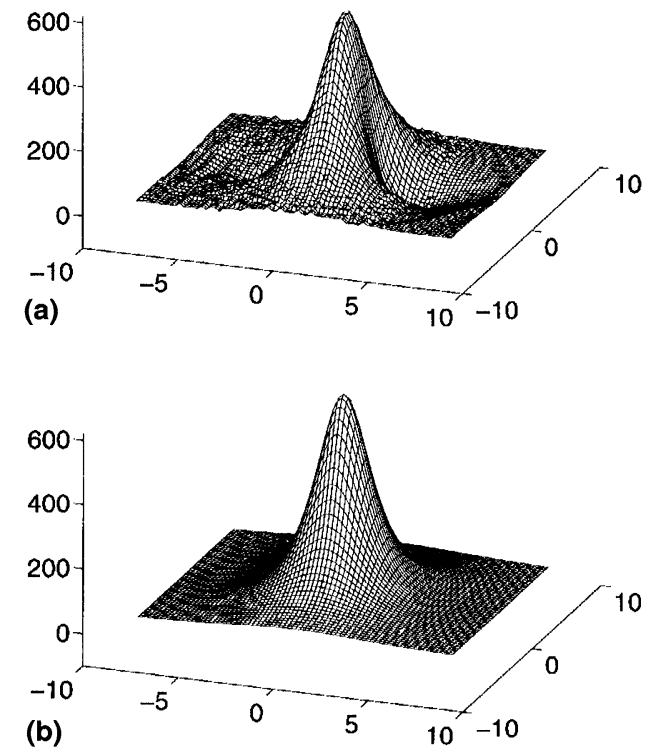

FIGURE $4 E_{\theta}$ : (a) Near field radiation from the photoconducting structure found using FDTD. (b) Near field radiation from an ideal Hertzian dipole.
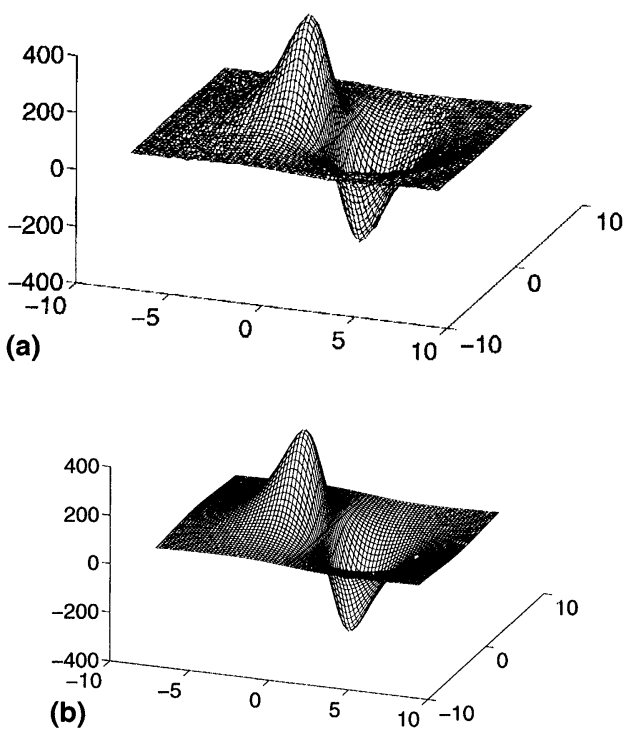

FIGURE $5 E_{R}$ : (a) Near field radiation from the photoconducting structure found using FDTD. (b) Near field radiation from an ideal Hertzian dipole.

simulation in Figures $4 \mathrm{a}$ and 5a. The effects of the various material parameters in the simulation space may be seen in Figure 4a. Specifically, the
GaAs/air interface causes some variation in the field distribution near the center of the surface.

To model the far field radiation, only terms which decrease as $1 / R$ are retained from Eq. (1). In this case, the electric field is given by:

$$
E_{\theta}(R, t)=\frac{\sin \theta}{4 \pi \varepsilon_{0} R c^{2}} \frac{\partial}{\partial t} \iiint_{V} J_{V} d V^{\prime}
$$

The far field radiation is proportional to the time derivative of the excitation. Eq. (2) is often used to approximate the far field radiation arising from photoconducting antennas and arrays [1]. However, (2) assumes a dipole source of negligible length and free space propagation. The use of (2) in the photo-conducting experiment also neglects the effects of the GaAs substrate and metal electrodes. In addition, numerical implementation of the time differentiation emphasizes the high frequency noise components. Figures $3 a$ and $3 b$ show a typical current pulse and two numerically smoothed versions of its derivative with respect to time. Smoothing is accomplished using a moving average filter. Figure $3 \mathrm{~b}$ compares to measured results such as [10].

It is expected that a more accurate representation of the far field radiation would be given with a near-to-far field transformation. This technique, which has been utilized by microwave and RF engineers for the analysis of radiation from, for example, horn antennas [11], utilizes an equivalent source methodology. The fields found on a closed virtual surface surrounding the source are used as new sources of radiation, replacing the original source, as illustrated in Figure 6. In this technique, the near field radiation arising from a complex structure may be calculated using the FDTD method, and the far field radiation may be found using the equivalent source in a homogeneous external problem space. Several formulations for an equivalent source representation exist, including vector forms such as the Schelkunoff equivalence theorem [12], the Stratton-Chu formulation [13], the Franz formulation [14], and the scalar Kirchhoff Surface Integral Representation, de- 


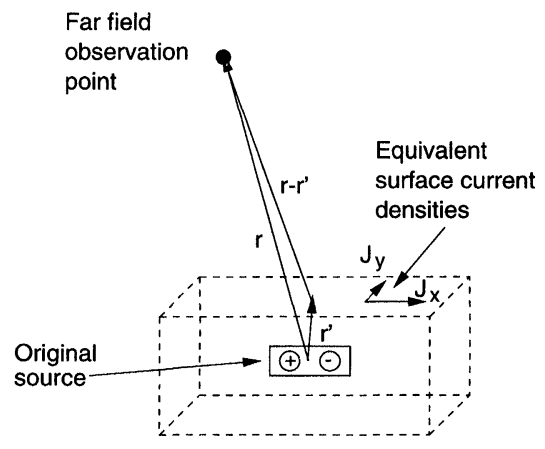

FIGURE 6 Equivalent current source model.

scribed, for example, in [15]. All provide a relationship between the electromagnetic fields on a surface surrounding a source and the field at a point outside the surface. The field produced by the equivalent source at the observation point will be the same as that produced by the original source. The Kirchhoff formulation would be the preferred technique in modeling the photo-conducting structure because the technique requires only the values of the electric fields on the equivalent surface. This is an advantage when used with the FDTD since the electric and magnetic fields are offset spatially by one half cell $[16,17]$. Additionally, since this is a scalar formulation, each field component may be calculated independently, allowing placement of the virtual surfaces to coincide with each field component's placement in the Yee cell. Application of this technique to the photoconducting structure is currently being implemented by the present authors.

\section{CONCLUSION}

Techniques for describing and modeling the terahertz radiation from a photo-conducting structure have been discussed. The physical source of radiation for laser excitation on a GaAs substrate was described, and an approximation of the source in terms of an ideal Hertzian dipole was presented. A simulation tool which couples a
Monte Carlo particle simulator with an FDTD electromagnetic solver was used to numerically determine the near field radiation. Computational results showing near field radiation patterns were shown. Techniques for approximating the far field radiation were discussed. Simulation results for a technique based on calculation of the derivative of the excitation were presented.

\section{Acknowledgement}

This work is supported by grant \#ECS-9312240 from the National Science Foundation. K. A. Remley is also supported under a National Science Foundation Graduate Research Fellowship.

\section{References}

[1] Froberg, N., Hu, B., Zhang, X. and Auston, D. H., "Terahertz radiation from a photoconducting antenna array”, IEEE J. Quantum Electron., 28, 2291-2301, Oct. 1992.

[2] Smith, P. R., Auston, D. H. and Nuss, M., "Subpicosecond photoconducting dipole antennas", IEEE J. Quantum Electron., 24, 255-260, Feb. 1988.

[3] Valdmanis, J. and Mourou, G., "Subpicosecond electrooptic sampling: principles and applications", IEEE $J$. Quantum Electron., QE-22, 69-78, Jan. 1986.

[4] El-Ghazaly, S. M., Joshi, R. P. and Grondin, R. O., "Electromagnetic and transport considerations in subpicosecond photoconductive switch model", IEEE Trans. Microwave Theory Tech., 38, 629-637, May 1990.

[5] Greene, B., Federici, J., Dykaar, D., Levi, A. and Pfeiffer, L., "Picosecond pump and probe spectroscopy utilizing freely propagating terahertz radiation", Opt. Lett., 16, 48-49, Jan. 1991.

[6] Goodnick, S., Pennathur, S., Ranawake, U., Lenders, P. and Tripathi, V. (1995). "Parallel implementation of a Monte Carlo particle simulation coupled to Maxwell's equations", Intl. J. of Num. Modelling, 8, pp. 205-219.

[7] Yee, K., "Numerical solution of initial boundary value problems involving Maxwell's equations in isotropic media", IEEE Trans. Antennas Propagat., AP-14, 302307, Dec. 1966.

[8] Berenger, J. P. (1994). "A perfectly matched layer for the absorption of electromagnetic waves",J. Computational Physics, 114, 185-200.

[9] Franceschetti, G. and Papas, C. H., "Pulsed Antennas", IEEE Trans. Antennas Propagat., AP-22, 651-661, Sept. 1974.

[10] Son, J., Sha, W., Norris, T., Whitaker, J. and Mourou, G., "Transient velocity overshoot dynamics in GaAs for electric fields > $200 \mathrm{kV} / \mathrm{cm} "$, Appl. Phys. Lett., 63, $923-$ 925, Aug. 1993. 
[11] Balanis, C. (1989). Advanced Engineering Electromagnetics. New York: Wiley.

[12] Schelkunoff, S. A. (1936). "Some equivalence theorems of electromagnetics and their application to radiation problems", Bell System Tech. J., 15, 92-112.

[13] Stratton, J. A., and Chu, L. J. (1939). "Diffraction Theory of Electromagnetic Waves", Phys. Rev., 56, 99-107.

[14] Franz, V. W. (1948). "Zur Formulierung des Huygensschen Prinzips", Z. Naturforsch. A, 3a, 500-506.

[15] Jackson, J. D. (1962). Classical Electrodynamics. New York: Wiley.

[16] De Moerloose, J. and Dezutter, D. "Surface Integral Representation Radiation Boundary Condition for the FDTD Method", IEEE Trans. Antennas Propagat., 41, $890-896$, July 1993.

[17] Ramahi, O. "Near- and Far-Field Calculation in FDTD Simulations Using Kirchhoff Surface Integral Representation", IEEE Trans. Antennas Propagat., 45, 753-759, May 1997.

\section{Authors' Biographies}

Kate Remley was born in Ann Arbor, Michigan in December, 1959. She worked as a broadcast engineer in Eugene, Oregon between 1983 and 1992, and was Chief Engineer of an AM/FM broadcast station from 1989-1992. She received the B.S. degree magna cum laude from Oregon State University in 1993 and the M.S. degree in 1995. She is currently working toward the Ph.D. degree at Oregon State University as a National Science Foundation Graduate Research Fellow. Her research interests include computational modeling and measurement of guided-wave optical, microwave, and millimeter-wave devices, modeling of quantum waveguide structures, and the theory of semiconductor device fabrication technology.

Andreas Weisshaar received the Diplom-Ingenieur (Dipl.-Ing.) degree in electrical engineering from the University of Stuttgart, Germany, in 1987, and the M.S. and Ph.D. degrees in electrical and computer engineering from Oregon State University in 1986 and 1991, respectively. Since 1992 he has been an Assistant Professor at the Department of Electrical and Computer Engineering at Oregon State University. His current research interests are in the areas of CAD of microwave circuits and components, interconnects and packaging, fiber optic sensors, and wireless communications.

Vijai K. Tripathi received the B.Sc degree from Agra University, India, M.Sc Tech. degree in electronics and radio engineering from Allahabad University, India and the M.S.E.E. and Ph.D. degrees in electrical engineering from the University of Michigan, Ann Arbor, in 1958, 1961, 1964 and 1968, respectively. He is a Professor and Head of Electrical and Computer Engineering at Oregon State University in Corvallis, Oregon. Prior to joining Oregon State in 1974, he had been with the Indian Institute of Technology in Bombay, the University of Michigan in Ann Arbor and the University of Oklahoma in Norman, Oklahoma. Over the years he has been a consultant to many industrial organizations including AVANTEK, EEsof Inc., Teledyne MMIC and Tektronix. His research activities are in the general areas of microwave and distributed parameter circuits and systems, computations electromagnetics, electronic packaging and interconnects.

Stephen M. Goodnick received his B.S. degree in Engineering Science from Trinity University in 1977, and his M.S. and Ph.D. in Electrical Engineering from Colorado State University in 1979 and 1983 respectively. He was an Alexander von Humboldt Fellow at the Technical University of Munich, Germany and the University of Modena, Italy in 1985 and 1986. He was the Melchor visiting chair at the University of Notre Dame in 1991. He was a faculty member from 1986 to 1997 in the Department of Electrical and Computer Engineering at Oregon State University, Corvallis, Oregon, where he held the rank of Professor. He is presently Chair and Professor of Electrical Engineering at Arizona State University. 

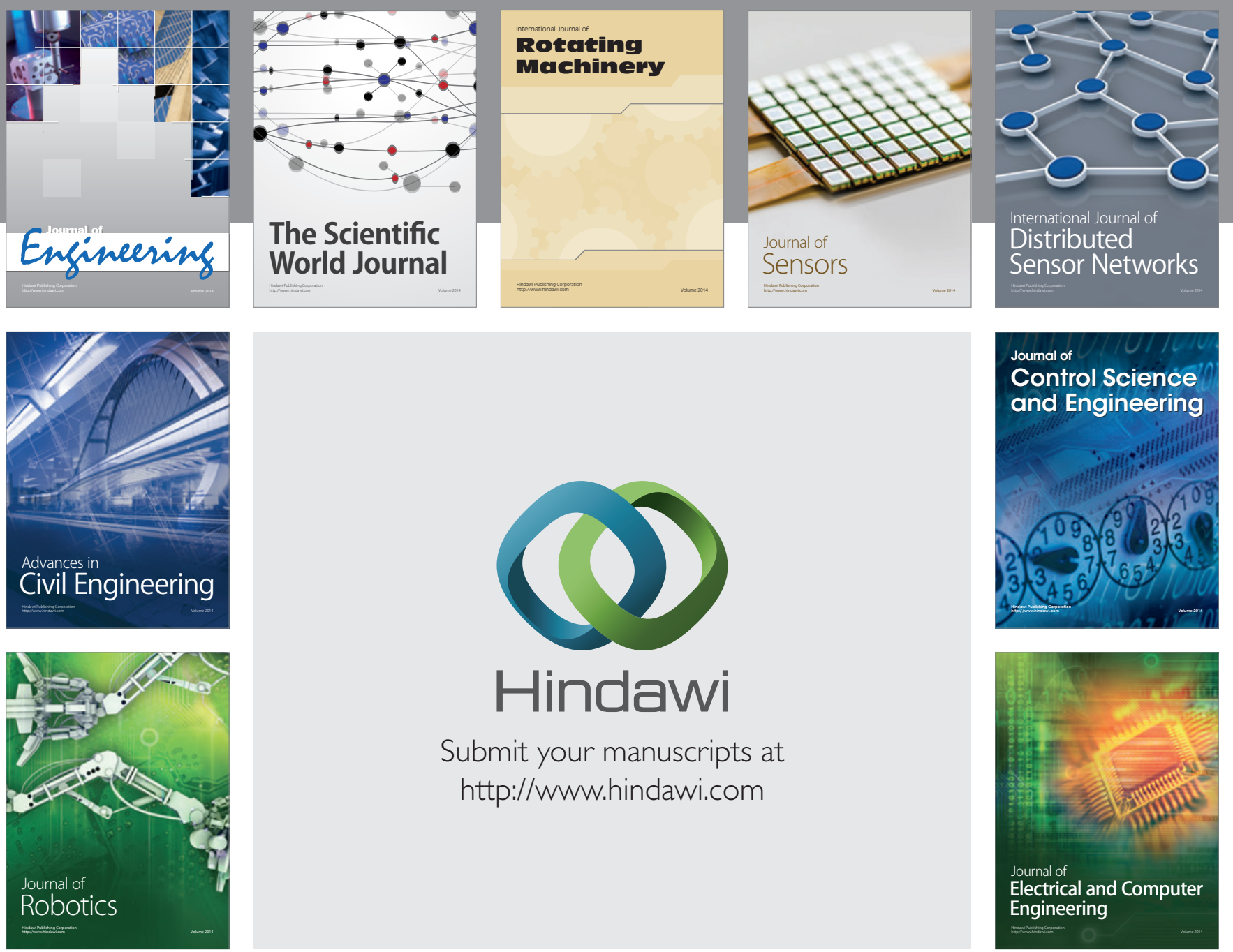

Submit your manuscripts at

http://www.hindawi.com
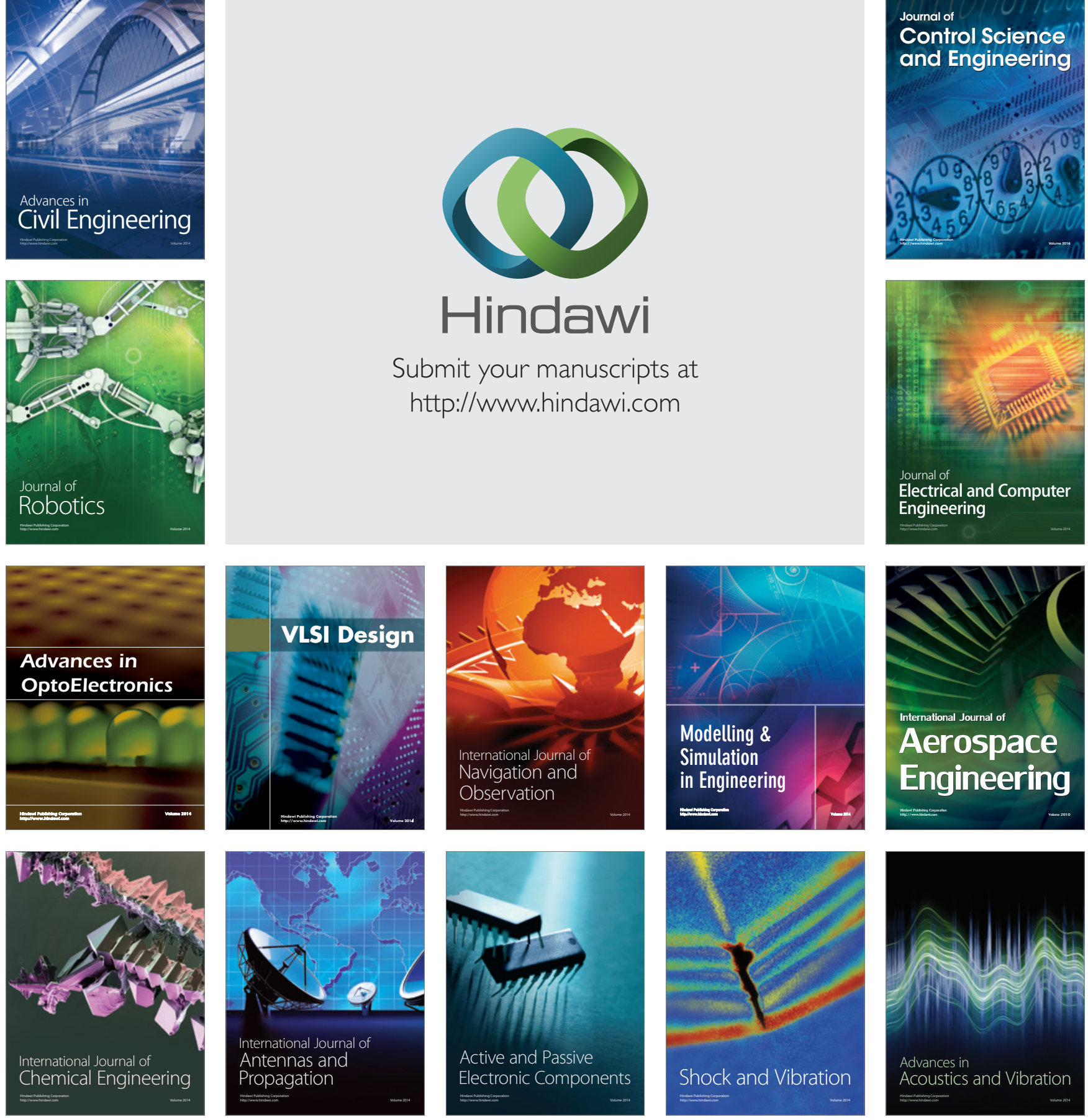